\title{
ESTUDO DAS CARACTERÍSTICAS DE COMBUSTÃO DE RESÍDUOS SÓLIDOS DA REGIÃO NORDESTE UTILIZANDO ANÁLISE TERMOGRAVIMÉTRICA
}

\author{
J. O. B. LIRA ${ }^{1}$, K. C. G. da SILVA ${ }^{2}$ e S. L. F. ANDERSEN ${ }^{2}$ \\ ${ }^{1}$ Universidade Federal da Paraíba, Departamento de Engenharia Química \\ ${ }^{2}$ Universidade Federal da Paraíba, Departamento de Energias Alternativas e Renováveis \\ E-mail para contato: silvia@cear.ufpb.br
}

\begin{abstract}
RESUMO - As exigências ambientais vêm aumentando a cada ano e impondo a necessidade de otimização dos processos industriais e gestão de resíduos. A utilização de resíduos sólidos como fonte de energia torna-se atrativa e diminui problemas com a disposição dos mesmos. O objetivo deste trabalho foi estudar o potencial energético e o comportamento da combustão de resíduos sólidos da região Nordeste do Brasil. Os resíduos (biomassas) selecionados foram: fibra da casca de coco, serragem de madeira (Massaranduba), bagaço de caju e bagaço de cana-de-açúcar. A caracterização físicoquímica das biomassas foi realizada por meio de análise imediata e estudo do comportamento térmico por análise termogravimétrica (TGA/DTG). A análise TGA/DTG foi realizada em atmosfera oxidante em condições dinâmicas de temperatura de 30 a $900{ }^{\circ} \mathrm{C}$ a uma taxa de aquecimento de $10{ }^{\circ} \mathrm{C} \cdot \mathrm{min}^{-1}$. Foram identificados picos de perda de massa referentes à umidade, devolatilização e oxidação. A caracterização das cinzas foi realizada por meio de análise de fluorescência de raios X e a possibilidade de fusão das cinzas foi determinada. Os resíduos bagaço de cana e casca de coco verde apresentam menor possibilidade de fusão das cinzas em processos de combustão, enquanto o bagaço de caju apresentou maior possibilidade A casca de coco foi a biomassa mais reativa apresentando menor $\mathrm{T}_{\mathrm{p}}$ com maior taxa de perda de massa (acima de 6,0 mg. $\mathrm{min}^{-1}$, $317,1^{\circ} \mathrm{C}$ ), porém, todos os resíduos estudados apresentaram bom potencial para aplicação como fonte alternativa de energia em processos de combustão.
\end{abstract}

\section{INTRODUÇÃO}

A demanda por energia é uma preocupação mundial. Estimativas da escassez do petróleo e as crescentes preocupações ambientais são fatores que desencadearam o interesse cada vez maior por fontes energéticas renováveis para substituir os combustíveis fósseis. Segundo o Balanço Energético Nacional (Brasil, 2013), o Brasil possui potencial para a produção de energia alternativa com participação de $42,4 \%$ em comparação a $13,2 \%$ da média mundial.

Utilizar a biomassa como fonte de energia é uma alternativa viável, pois existe em abundância, é de baixo custo e fácil acesso e, além disso, o Brasil possui condições climáticas e geográficas favoráveis para a sua produção (Figueiredo, 2011). Parte da demanda energética 


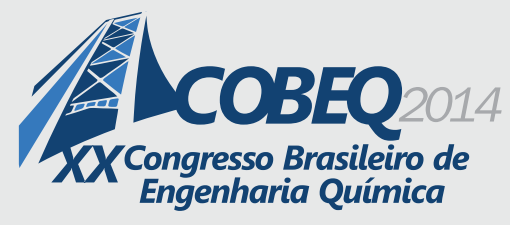

19 a 22 de outubro de 2014

Florianópolis/SC

brasileira ainda é atendida pela queima de madeira. Do total, aproximadamente $60 \%$ é desperdiçado nas serrarias durante o processamento primário, e $20 \%$ são desperdiçados no processamento secundário (Brasil, 2009).

O Brasil é considerado o maior produtor de cana-de-açúcar do mundo com cerca de 4,5 milhões de hectares plantados. Na safra de 2001, por exemplo, o Brasil processou 270 milhões de toneladas de cana-de-açúcar para a produção de açúcar e etanol, gerando aproximadamente 11 milhões de toneladas de bagaço. O bagaço da cana de açúcar pode ser, e é utilizado como fonte de energia sendo a quantidade média de energia produzida por cada tonelada de $100 \mathrm{kWh}$, (Grimoni, Galvão e Udaeta, 2004). No caso do Estado da Paraíba, o bagaço da cana-de-açúcar é responsável pela geração do maior volume de resíduos sólidos do estado. Em 2002, foi realizado um levantamento dos resíduos sólidos no estado visando o cumprimento com a Política Nacional de Resíduos Sólidos. Foi contabilizada a geração de cerca de 1.365.230 toneladas, o que correspondeu a $60,7 \%$ de todo o resíduo sólido produzido no território paraibano (SUDEMA, 2004).

O caju, fruto originalmente brasileiro, representa 764.472 hectares ou $24,4 \%$ de área plantada no território brasileiro, e teve produção de 2.077.065 toneladas em 2011 (ADECE, 2013). A região Nordeste é a maior produtora de caju, o qual se destacam os estados do Ceará, Rio Grande do Norte, Piauí e Bahia como os principais produtores. Deste fruto, a parte de maior valor comercial é a castanha, enquanto que o pseudofruto é na maioria das vezes utilizado para fazer sucos e polpas gerando assim grandes quantidades de resíduos sólidos (bagaço), que geralmente é descartado ou utilizado para complemento de ração animal. O bagaço de caju representa aproximadamente $20 \%$ em peso do pedúnculo, e até o ano de 2007, eram gerados, em média, 300.000 toneladas desse resíduo por ano no Brasil (Santos et al., 2007).

O Brasil possui cerca de 280 mil hectares cultivados com coqueiro, distribuídos, praticamente por todo o território nacional, com produção de 1.962.434 toneladas do fruto. O seu cultivo é predominante na faixa litorânea, especialmente nas regiões Nordeste e Norte, sendo os maiores produtores a Bahia, Ceará, Sergipe e Pará (ADECE, 2013). A casca do coco representa até $80 \%$ do peso final do fruto e estima-se que $70 \%$ do lixo recolhido nas praias brasileiras são compostas de casca de coco verde (A lavoura, 2012). Gerando um grande problema ambiental, pois as cascas são levadas para lixões e outras áreas consideradas inadequadas, contribuindo para ampliar os problemas de resíduos sólidos urbanos (Bitencourt, Pedrotti, 2008).

A maior parte da energia consumida no mundo vem do processo de combustão que é utilizada em meios de transporte, usinas termelétricas, processos industriais, aquecimento doméstico, geradores, cozimento de alimentos, entre outros. A utilização do processo de combustão para o aproveitamento energético da biomassa já é conhecido, e um exemplo típico são as fornalhas a lenha das caldeiras. Sendo assim, é interessante estudar o potencial de utilização dos resíduos supracitados como fonte alternativa de energia utilizando processo de combustão. 


\section{9 a 22 de outubro de 2014 \\ Florianópolis/SC}

O objetivo deste trabalho foi estudar as características de combustão de resíduos sólidos comuns na região Nordeste do Brasil, para avaliação do potencial de utilização dos mesmos como fonte alternativa de energia. Os resíduos selecionados neste estudo foram: casca de coco verde, serragem de madeira (Massaranduba), bagaço de caju e bagaço de cana-de-açúcar. As amostras foram caracterizadas físico-quimicamente utilizando as técnicas de análise imediata e análise termogravimétrica (TGA) para o estudo do comportamento da combustão. As cinzas das amostras também foram caracterizadas quimicamente para estudo da possibilidade de fusão das mesmas, utilizando a técnica de fluorescência de raios X (FRX).

\section{MATERIAL E MÉTODOS}

Para este estudo foram selecionadas quatro biomassas (resíduos sólidos): bagaço da canade-açúcar (indústria sucroalcoolera), casca do coco verde (resíduo recolhido na praia de Tambaú), serragem de madeira (Massaranduba - madeireira) e bagaço de caju (indústria de poupa de frutas). Todas as amostras foram coletadas entre novembro de 2013 e fevereiro de 2014 em estabelecimentos da região da cidade de João Pessoa - PB.

As biomassas foram caracterizadas por meio de análise imediata para a determinação dos teores de umidade, cinzas, material volátil e carbono fixo, conforme as normas ASTM (E871-82, D1102-84 e E872-82). O teor de carbono fixo foi calculado por diferença. O teor de umidade do bagaço de caju não foi determinado pois a amostra foi recebida previamente seca. O poder calorífico (PC) foi calculado com base numa equação empírica (DEMIRBAS, 1997) a partir da análise imediata, no qual a equação é função do teor de carbono fixo (FC) e o teor do material volátil (VM), conforme equação 1 .

$\mathrm{PC}=0,312(\mathrm{FC})+0,1534(\mathrm{VM}) \quad\left[\mathrm{MJ} \cdot \mathrm{kg}^{-1}\right]$

Para o estudo do comportamento da combustão dos resíduos, utilizou-se análise termogravimétrica (TGA) e termogravimetria diferencial (DTG), utilizando o equipamento Shimadzu, DTG 60H, atmosfera oxidante (ar sintético, 99,99\%), a uma vazão de 50 mL. min $^{-1}$. O programa de temperatura foi de 30 até $900{ }^{\circ} \mathrm{C}$ com taxa de aquecimento de $10^{\circ} \mathrm{C} \cdot \mathrm{min}^{-1}$. A massa das amostras foi de aproximadamente $10 \mathrm{mg}$ em cadinho de alumina.

Para a caracterização química das cinzas, as mesmas foram obtidas em forno mufla a $575{ }^{\circ} \mathrm{C}$ durante pelo menos $2 \mathrm{~h}$. Para a avaliação da composição química das cinzas foi utilizada a técnica de FRX, utilizando equipamento Shimadzu, modelo EDX-820, massa de amostra aproximadamente $300 \mathrm{mg}$ e porta amostra de polietileno. Caracterizar quimicamente as cinzas da biomassa é importante visto que a composição mineral pode causar diversos problemas de operação dos reatores de conversão termoquímica, especialmente por ter possível ponto de fusão relativamente baixo $\left(<800{ }^{\circ} \mathrm{C}\right)$. Pronobis, 2005, propôs uma correlação baseada na composição química das cinzas de combustíveis para verificar a possível fusão das mesmas. Essa correlação foi utilizada neste trabalho para avaliar este fenômeno e está apresentada na equação 2. Quanto maior o B/A $(+P)$, maior a tendência de fusão, ou menor a temperatura de fusão (Pronobis, 2005; Virmond, et al., 2012). 
$B / A_{(+P)}=\left[\frac{\mathrm{Fe}_{2} \mathrm{O}_{3}+\mathrm{CaO}+\mathrm{MgO}+\mathrm{Na}_{2} \mathrm{O}+\mathrm{K}_{2} \mathrm{O}+\mathrm{P}_{2} \mathrm{O}_{5}}{\mathrm{SiO}_{2}+\mathrm{Al}_{2} \mathrm{O}_{3}+\mathrm{TiO}_{2}}\right]$

\section{RESULTADOS E DISCUSSÃO}

\subsection{Caracterização físico-química dos resíduos}

A Tabela 1 mostra os resultados da caracterização físico-química das biomassas e caracterização química das cinzas.

Tabela 1 - Caracterização físico-química das biomassas e cinzas das biomassas.

\begin{tabular}{|c|c|c|c|c|}
\hline Amostra & $\begin{array}{c}\text { Bagaço de } \\
\text { Cana-de-açúcar }\end{array}$ & Bagaço de Caju & $\begin{array}{c}\text { Serragem de } \\
\text { Massaranduba }\end{array}$ & $\begin{array}{c}\text { Casca de Coco } \\
\text { Verde }\end{array}$ \\
\hline Umidade $^{*}$ & 9,17 & $\mathrm{NA}^{\mathrm{c}}$ & 17,05 & 11,97 \\
\hline Material Volátil *,a & 83,10 & 75,23 & 83,47 & 75,31 \\
\hline Cinzas $^{*, a}$ & 5,98 & 1,93 & 0,598 & 7,23 \\
\hline Carbono Fixo *,a & 11,00 & 22,83 & 15,93 & 17,46 \\
\hline PCS $^{\mathbf{b}}$ & 16,18 & 18,66 & 17,77 & 17,00 \\
\hline \multicolumn{5}{|c|}{ Composição das cinzas * } \\
\hline $\mathrm{SiO}_{2}$ & 57,919 & $<\mathrm{LD}$. & 9,069 & 33,814 \\
\hline $\mathrm{K}_{2} \mathrm{O}$ & 17,171 & 78,749 & 10,307 & 5,111 \\
\hline $\mathrm{CaO}$ & 10,839 & 3,880 & 77,516 & 43,415 \\
\hline $\mathrm{P}_{2} \mathrm{O}_{5}$ & 7,758 & 13,407 & $<\mathrm{LD}$. & $<\mathrm{LD}$. \\
\hline $\mathrm{SO}_{3}$ & 3,083 & 2,836 & 2,122 & 3,573 \\
\hline $\mathrm{Fe}_{2} \mathrm{O}_{3}$ & 2,005 & 0,545 & 0,795 & 13,141 \\
\hline $\mathrm{MnO}$ & 0,645 & 0,225 & 0,061 & 0,122 \\
\hline $\mathrm{TiO}_{2}$ & 0,262 & $<\mathrm{LD}$. & $<\mathrm{LD}$. & 0,077 \\
\hline $\mathrm{Cr}_{2} \mathrm{O}_{3}$ & 0,127 & $<\mathrm{LD}$. & 0,043 & $<\mathrm{LD}$. \\
\hline $\mathrm{ZnO}$ & 0,112 & 0,158 & 0,024 & 0,189 \\
\hline $\mathrm{CuO}$ & 0,059 & 0,162 & $<$ LD. & 0,066 \\
\hline $\mathrm{V}_{2} \mathrm{O}_{5}$ & 0,020 & $<\mathrm{LD}$. & $<$ LD. & 0,480 \\
\hline $\mathrm{NiO}$ & $<\mathrm{LD}$. & 0,024 & 0,044 & $<\mathrm{LD}$. \\
\hline $\mathrm{B} / \mathrm{A}_{(+\mathrm{P})}$ & 0,67 & Muito elevado & 9,92 & 1,54 \\
\hline
\end{tabular}

${ }^{*} \% \mathrm{~m} / \mathrm{m}$. ${ }^{a}$ base seca e livre de cinzas. ${ }^{b}$ calculado $\left[\mathrm{MJ} \mathrm{kg}^{-1}\right.$ ] (Equação 1). ${ }^{c}$ não analisado. L.D. - Limite de detecção.

Observa-se que os teores de material volátil, entre 75,23 e $83,47 \%$, são elevados o que indicam uma possível combustão rápida. É comum esses teores serem elevados em biomassas e sugerem reatividade elevada desse tipo de combustível (Virmond, et al. 2012). Resultados similares para os teores de material volátil podem ser encontrados na literatura, como por exemplo: 85,97\% para o bagaço de cana-de-açúcar e (Nhuchhen, Salam, 2012), 85,73\% para o bagaço de caju (Wilson, et al., 2011), 83,5\% para a serragem de madeira (García, et al., 2014). O 


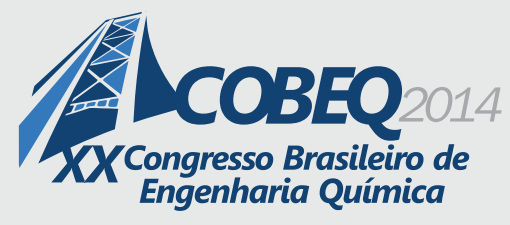

19 a 22 de outubro de 2014

Florianópolis/SC

poder calorífico superior (PCS) foi calculado e os resultados foram entre 16,18 e 18,66 ${\mathrm{MJ} . \mathrm{kg}^{-1}}^{-1}$ que podem ser comparados com os encontrados experimentalmente por outros autores. Um exemplo é o bagaço de cana que experimentalmente apresentou 17,7 MJ.kg ${ }^{-1}$ (Demirbas, 2004)

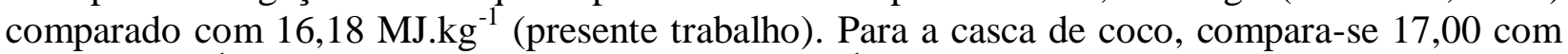
20,51 MJ.kg-1 (Tsamba, Yang, 2006) e 22,38 MJ.kg-1 para o bagaço de caju (Wilson et al, 2011) que calculado neste trabalho apresentou $18,66 \mathrm{MJ}_{\mathrm{kg}}{ }^{-1}$, indicando tanto uma boa possibilidade de aproveitamento desses resíduos como fonte de energia, como valida a utilização da equação 1 para estimar o poder calorífico superior assim como conclui García, et al., 2014.

O teor de cinzas da serragem de madeira é inferior às demais biomassas analisadas. Este teor pode variar dependendo da safra e local de obtenção das mesmas, um exemplo é o bagaço de cana de açúcar que apresentou teor de aproximadamente $6 \%$ em massa e na literatura encontramse valores de 0,69 até $10 \%$ m/m (Virmond, et al. 2012, De Paula, et al., 2009).

Observando a composição química das cinzas, os principais elementos inorgânicos são Si, $\mathrm{Ca}, \mathrm{K}$ e Fe. O bagaço de cana-de-açúcar e a casca de coco verde apresentam os maiores valores de sílica enquanto que o bagaço de caju apresentou quantidade inferior ao limite de detecção do equipamento, no entanto apresentou elevado teor de $\mathrm{K}_{2} \mathrm{O}(78,75 \%)$. A presença de $\mathrm{K}$ nas cinzas pode ocasionar problemas pois forma compostos de menor temperatura de fusão facilitando assim a deposição de cinzas nas caldeiras e/ou demais reatores de conversão térmica causando a redução à transferência de massa e consequentemente a diminuição da eficiência do equipamento. Sendo assim, processos com temperatura mais baixa ou a possibilidade de co-combustão com outro combustível de maior temperatura de fusão das cinzas é desejável quando se deseja utilizar o bagaço de caju.

$\mathrm{O}$ índice $\mathrm{B} / \mathrm{A}_{(+\mathrm{P})}$ foi calculado e verifica-se que os resíduos bagaço de cana e casca de coco verde apresentam menor possibilidade de fusão das cinzas em processos de combustão, enquanto o bagaço de caju confirmou que possui maior possibilidade devido aos elevados teores de metais alcalinos e alcalino-terrosos. A serragem de madeira também apresentou tendência de fusão provavelmente pela presença de cálcio.

\subsection{Comportamento da combustão}

O resultado do comportamento dos resíduos em processo de combustão é mostrado na Figura 1. Os termogramas obtidos do TGA/DTG são importantes para a compreensão de como ocorre a combustão de materiais orgânicos e/ou combustíveis. Regiões com perda de massa foram observadas, representando as etapas de secagem, devolatilização e oxidação (regiões comuns em processos de combustão). A região de secagem deve-se à liberação de umidade (temperaturas inferiores a $100{ }^{\circ} \mathrm{C}$ ). O segundo pico é geralmente o maior pico e representa a liberação do material volátil, sendo assim, também indica a temperatura ignição $\left(\mathrm{T}_{\mathrm{ig}}\right)$. Essa é uma região de queima rápida e a perda de massa pode atingir seu maior valor. A Figura 1 também mostra os dados de taxa máxima de queima e temperatura de pico $\left(\mathrm{T}_{\mathrm{p}}\right)$ das biomassas obtidas a partir da análise de TGA/DTG. Para a discussão desses resultados foi desconsiderado o pico 
referente à perda de umidade. Os demais picos correspondem à combustão de matéria orgânica mais pesada e carbono fixo, e estão numerados para melhor entendimento.
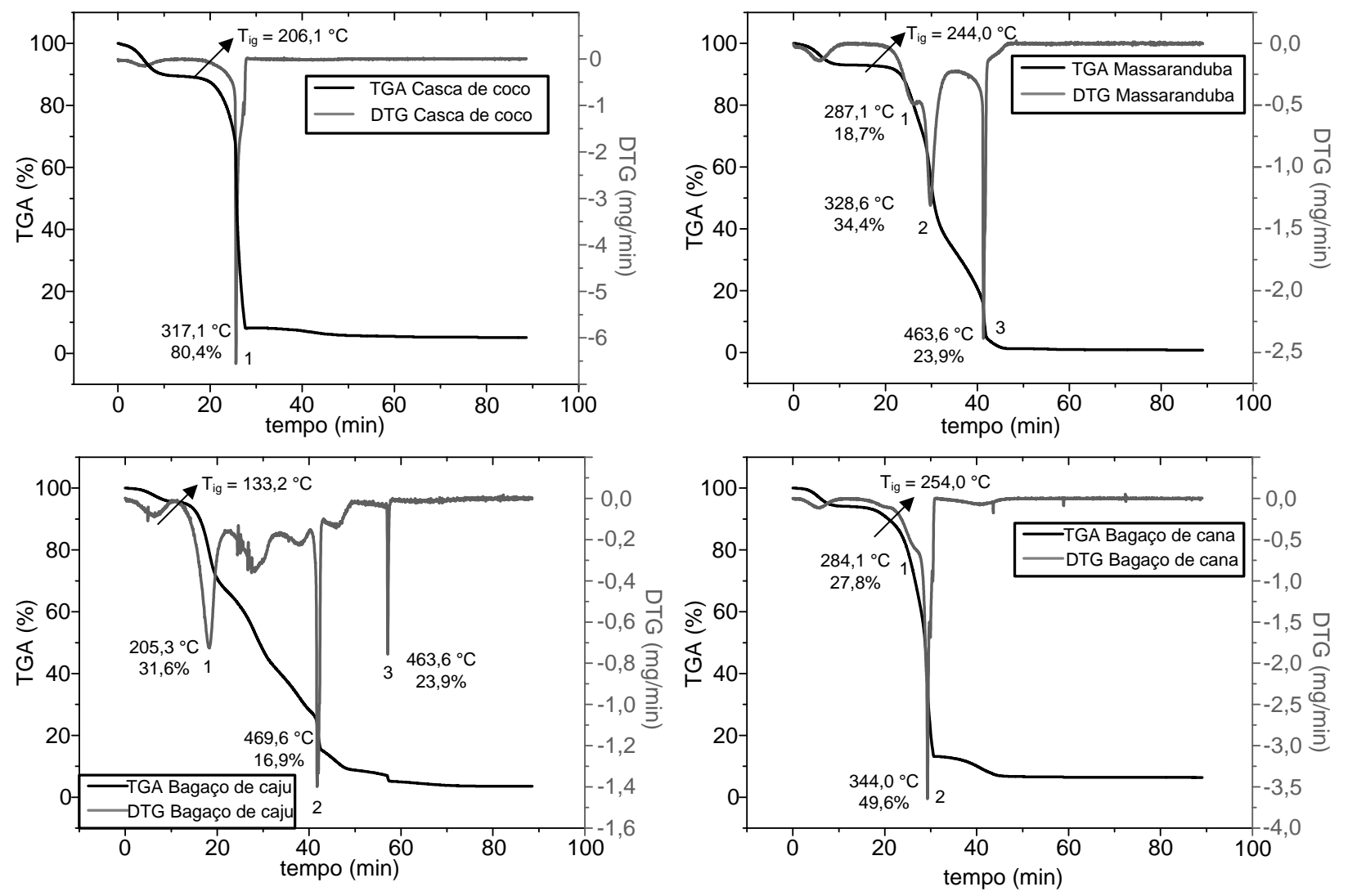

Figura 1 - TGA/DTG das amostras.

O resultado da análise TGA/DTG, quando realizada em atmosfera oxidante as perdas de massa são relacionadas à liberação de umidade e material volátil seguida de oxidação homogênea do mesmo e heterogênea do carbono fixo.

Observa-se que cada amostra apresentou um comportamento diferente com distintos números de picos referentes às perdas de massa. A casca de coco apresentou um pico de liberação de umidade e outro representando os fenômenos de liberação de material volátil e queima rápida do mesmo juntamente com o carbono fixo. A $\mathrm{T}_{\mathrm{p}}$ foi de $317,1{ }^{\circ} \mathrm{C}$ com perda de massa de $80,4 \%$. Similarmente, o bagaço de cana apresentou somente um pico, porém com certa distorção no início, sendo comum visto as diferentes composições químicas. Observa-se então que a $\mathrm{T}_{\mathrm{p}}$ do maior pico é $344^{\circ} \mathrm{C}$ e é o pico referente à maior taxa de perda de massa (aprox. 3,5 mg. mim $^{-1}$ ). Zhang et al., 2014, caracterizaram grama utilizando a termogravimetria. Os autores identificaram picos referentes à decomposição de celulose e lignina apresentando o mesmo comportamento das biomassas estudadas neste trabalho. Sendo assim, o comportamento é característico de biomassas lignocelulósicas. 
As amostras de serragem de madeira e bagaço de caju apresentaram mais picos referentes a perdas de massa, o que é comum devido à diferenças de estrutura física e química particular de cada amostra. Os picos em temperaturas mais elevadas, acima de $400{ }^{\circ} \mathrm{C}$, são provavelmente referentes à queima do carbono fixo sendo às anteriores referentes à liberação e oxidação do material volátil.

A $T_{\text {ig }}$ das amostras foi determinada como sendo a temperatura em que o pico de liberação do material volátil inicia. Esses resultados, juntamente com $\mathrm{T}_{\mathrm{p}}$ e perda de massa referente a cada pico também podem ser observados na Figura 1.

A comparação de taxas de queima torna-se difícil visto que a composição e origem das biomassas são bastante variadas, somadas ao fato de serem resíduos. As $\mathrm{T}_{\mathrm{ig}}$ variaram entre $133,2{ }^{\circ} \mathrm{C}$ (bagaço do caju) e $254^{\circ} \mathrm{C}$ (bagaço de cana). Sendo assim, bagaço do caju é mais fácil de inflamar comparadas as outras biomassas. Para a maioria das amostras é observado que a maior perda de peso ocorre em torno dos $330{ }^{\circ} \mathrm{C}$, com exceção do bagaço caju que ocorreu a uma temperatura de $205^{\circ} \mathrm{C}$. É comum que a máxima taxa de perda de massa seja o pico 1, porém tanto para o bagaço de caju quanto para a serragem de madeira o pico 2 e 3 apresentaram maior taxa de queima, respectivamente. Considerando mais reativa a biomassa com maior taxa de perda de massa em menor temperatura, verifica-se que a casca de coco verde é o resíduo mais reativo com taxa máxima de perda de massa superior a $6 \mathrm{mg} \cdot \mathrm{min}^{-1}$ a $317^{\circ} \mathrm{C}$.

Outros trabalhos na literatura apresentaram resultados similares quando analisados resíduos e/ou biomassa e seu comportamento durante processo de combustão. Nos perfis TGA/DTG foram apresentados as três fases correspondentes a secagem, devolatilização e oxidação e as temperaturas de ignição correspondente à temperatura do pico de devolatização foram em torno de 124 a $250{ }^{\circ} \mathrm{C}$ para resíduos sólidos agroindustriais (Virmond, et al., 2012), 200 a $360{ }^{\circ} \mathrm{C}$ para misturas de linhito e resíduos de azeitona (Yuzbasi, 2011), 200 a $300{ }^{\circ} \mathrm{C}$ (Idris, Rahman e Ismail, 2012) para Palma.

\section{CONCLUSÃO}

Neste trabalho foram selecionados quatro resíduos sólidos, que foram caracterizados físicoquimicamente para aplicações como fonte de energia em processos de combustão. Os resíduos estudados foram: serragem de madeira, casca de coco, bagaço de caju e bagaço de cana-deaçúcar.

Os resíduos apresentaram teores de cinzas entre 0,6 e 7,23\% em massa, o que sugere potencial de redução de volume, para a disposição, quando submetidos à combustão. $\mathrm{O}$ estudo do comportamento da combustão utilizando análise termogravimétrica mostrou que os resíduos apresentaram $\mathrm{T}_{\mathrm{ig}}$ variando entre 133,2 e $254^{\circ} \mathrm{C}$, para o bagaço do caju e o bagaço de cana respectivamente. A casca de coco foi a biomassa mais reativa apresentando menor $\mathrm{T}_{\mathrm{p}}$ com maior taxa de perda de massa (acima de $6,0 \mathrm{mg} \cdot \mathrm{min}^{-1}, 317,1{ }^{\circ} \mathrm{C}$ ). O comportamento da combustão mostrou distintos picos referentes à liberação de materiais volátil, oxidação homogênea do mesmo e oxidação heterogênea do carbono fixo. 
A composição química das cinzas dos resíduos e o calculo do potencial de fusão mostrou que o bagaço de cana e a casca de coco apresentaram menor potencial para a fusão das cinzas em temperaturas elevadas, enquanto que o bagaço de caju e a serragem de madeira apresentaram maiores teores de metais alcalinos e alcalino-terrosos, destacando o $\mathrm{K}$ e $\mathrm{o} \mathrm{Ca}$, que são responsáveis pela diminuição da temperatura de fusão. Neste caso, recomenda-se um estudo para utilização desses resíduos em processos de conversão térmica utilizando temperaturas inferiores ou em processos de co-combustão com combustíveis no qual as cinzas possuem maior ponto de fusão.

Em suma, todos os resíduos estudados apresentam potencial para utilização como fonte de energia, tanto pela disponibilidade dos mesmos, quanto pelo seu comportamento em processos de combustão, porém os que melhor seriam aplicados diretamente são a casca de coco e o bagaço de cana, tanto pela reatividade quanto pelo menor potencial de fusão das cinzas.

\section{AGRADECIMENTOS}

Os autores agradecem o CNPq pelo apoio financeiro e aos laboratórios: LSR (CT - UFPB), LABEME (CT - UFPB), e Laboratório de Tecnologia Ambiental - NUPPRAR/UFRN.

\section{REFERÊNCIAS}

ADECE. Agência de Desenvolvimento do Estado do Ceará. Perfil da produção de frutas. p. 32, 2013

Aproveitamento de resíduos. Coco: Qual o destino dar a casca? A Lavoura, n. 690, p. 26-30, 2012.

BITENCOURT, D. V.; PEDROTTI, A. Uso da casca de coco: Estudo das viabilidades de implantação de usina de beneficiamento de fibra de coco em Sergipe. Revista da Fapese, v.4, n.2, p. 113-112, 2008.

BRASIL. Ministério de Minas e Energia. Balanço energético nacional 2013. Brasília: MME, p. 21, 2013.

DEMIRBAS, A. Calculation of higher heating values of biomass fuels. Fuel, v. 76, n. 5, p. 431434, 1997.

DEMIRBAS, A. Combustion characteristics of different biomass fuels. Progress in energy and combustion Science, v.30, p. 219-230, 2004.

FIGUEIREDO, A. L., Pirólise termoquímica de pós da fibra de coco seco em um reator de cilindro rotativo para produção de bio-óleo. 2011. 113 f. Dissertação de mestrado - Universidade Federal do Rio Grande do Norte, Natal, 2011.

GARCIA, R.; PIZARRO, C.; LAVIN, A. G.; BUENO, J. L. Spanish biofuels heating value 
estimation. Part II: Proximate analysis data. Fuel, v. 117, p. 1139-1147, 2014.

GRIMONI, J. A.; GALVÃO, L. C. R.; UDAETA, M. E. M. Iniciação a conceitos de sistemas energéticos para o desenvolvimento limpo. São Paulo: Editora Edusp, 2004.

HANZADE, H. Combustion characteristics of different biomass materials. Energy Conversion and Management, v. 44, p. 155-162, 2003.

IDRIS, S. S.; RAHMAN, N. A.; ISMAIL, K. Combustion characteristics of Malaysian oil palm biomass, sub-bituminous coal and their respective blends via thermogravimetric analysis (TGA). Bioresource Technology, v. 123, p. 581-591, 2012.

MAGDZIARZ, A.; WILK, M. Thermogravimetric study of biomass, sewage sludge and coal Combustion. Energy Conversion and Management, v. 75, p. 425-430, 2013.

NHUCHHEN, D.; SALAM, P. A. Estimation of higher heating value of biomass from proximate analysis: A new approach. Fuel, v. 99, p. 55-63, 2012.

SANTOS, R. P.; SANTIAGO, A. A. X.; GADELHA, C. A. A.; et al. Production and characterization of the cashew (Anacardium occidentale L.) peduncle bagasse ashes. Journal of Food Engineering, n. 79, p. 1432-1437, 2007.

SUDEMA, Inventário de resíduos sólidos industriais do estado da Paraíba - Brasil, p. 92, 04 mapas, 2004.

WILSON, L.; YANG, W.; BLASIAK,W.; et al, Thermal characterization of tropical biomass feedstocks. Energy Conversion and Management, v.52, p. 191-198, 2011.

YUZBASI, N. S; SELÇUK, N. Air and oxy-fuel combustion characteristics of biomass/lignite blends in TGA-FTIR. Fuel Processing Technology, v. 92, p. $1101-1108,2011$.

ZHANG, J.; FENG, L.; WANG, D.; ZHANG, R.; LIU, G.; CHENG, G. Thermogravimetric analysis of lignocellulosic biomass with ionic liquid pretreatment. Bioresource Technology, v. 153, p. 379-382, 2014. 